Case Report

\title{
Constrictive Myelopathy in An Old Giant Dog
}

\section{Omar Victor Del Vecchio*}

Centro Veterinario Caleidos, Via Lanfranco 4 zip code 17011, Albisola Superiore, SV, Italy

*Corresponding author: Omar V Del Vecchio, Centro Veterinario Caleidos, Via Lanfranco 4 zip code 17011, Albisola Superiore, SV, Italy, E-mail: omarvet@icloud.com, ORCID: https://orcid.org/0000-0002-7766-7327

Received date: 11 December, 2021 | Accepted date: 23 December, 2021 | Published date: 29 December, 2021

Citation: Del Vecchio OV. (2021) Constrictive Myelopathy in An Old Giant Dog J Clin Vet Res 1(1): doi https://doi.org/10.54289/ JCVR2100105

Copyright: () 2021 Del Vecchio OV. This is an open-access article distributed under the terms of the Creative Commons Attribution License, which permits unrestricted use, distribution, and reproduction in any medium, provided the original author and source are credited.

\begin{abstract}
In pugs, West Highland white terriers and other small breeds, congenital or acquired malformations/deviations of the spine are commonly reported, resulting, amongst other complications, in chronic meningeal compression and fibrosis, and interference with cerebrospinal fluid flow into the sub-arachnoid space. This leads to constrictive myelopathy $(\mathrm{CM})$ which involves the constriction and consequent reduced diameter of the spinal cord accompanied by parenchymal damage (mostly gliosis).

A11-year-old giant mixed breed male dog presented with ataxia in the hindlimbs, paresis, reduced proprioception, and normal reflexes. Neurolocalization was to the thoracolumbar spinal cord (T3-L3). Myelographic computed tomography and magnetic resonance imaging (MRI) of this tract showed spondylosis, multiple disc protrusions with mild cord compression, and reduced spinal cord diameter. Genetic test for SOD1 mutations performed to evaluate predisposition to degenerative myelopathy negative results; although poorly compressive, the symptoms were attributed to multiple disc protrusions, leading to spinal cord atrophy. The dog underwent physiotherapy and 23 months later, was more ataxic and paretic however still ambulatory; repeated MRI confirmed the previous findings, however, the onset of concomitant megacolon and a chronic kidney failure, induced the owner to euthanasia. Histopathological assessment indicated diffused remodeling of the meninges with progressive constriction of the spinal cord of concomitant megacolon and a chronic kidney failure, induced the owner to euthanasia. Histopathological assessment indicated diffused remodeling of the meninges with progressive constriction of the spinal cord. Altered distribution of the mechanical forces among the meningeal layers with stiffening of the ventral pachymeninges, probably caused by the disc protrusions, was considered the most likely trigger. To our knowledge, this is the first case reporting clinical, MRI and histological findings resembling an acquired constrictive myelopathy in a large-breed dog.
\end{abstract}

Keywords: Gliosis, Meninges, Myelopathy, Physiotherapy, Spondylosis

Abbreviation:CM: Constrictive Myelopathy, MRI: Magnetic Resonance Imaging, DMs: Degenerative Myelopathies, SOD1: Superoxide Dismutase, NSAID: Non-Steroidal Anti-Inflammatory Drug 


\section{Introduction:}

Chronic and progressive hind limb ataxia and paresis due to thoracolumbar spinal cord injuries are quite frequent in adult or senior giant dogs. This occurs because of several spinal cord diseases, such as chronic disc degeneration (disc protrusions), followed by degenerative myelopathies (DMs) and neoplasia [12]. Beside these three categories of spinal cord diseases, there is another type of degenerative disease affecting the spinal cord, called "constrictive" myelopathy (CM), which is usually reported in Pugs and in other small breed dogs [3-5]. To date, CM has not been reported in large breed dogs.

Thoracolumbar disc protrusions (Hansen 2) are more common in giant breeds, having an insidious onset, usually after 5-6 years of age, and tending to manifest clinically after that age [6].

Chronic spinal cord compression results from fibroid degeneration of the disc and weakening of the dorsal portion of the annulus fibrosus, which finally protrudes into the spinal canal [7]. It can also occur in the cranial thoracic tract resulting in a reduced resistance of the intercapital ligament [8]. This condition leads to slow but progressive spinal cord compression, with demyelination of the white matter and consequent appearance of hind limb ataxia and paresis. Usually, the affected dogs show pain when the thoracolumbar spine is pressed; however, in some cases the pain in the spinal cord abates. Therefore, pain may not be the most relevant symptom.

We also noted concomitant ventral spondylosis, which was not related to disc protrusions or clinical alterations. DM is a neurodegenerative disease of the spinal cord that usually starts in the thoracolumbar tract of adult/senior dogs. It causes demyelination and axonal degeneration mainly in the dorsolateral portion of the white matter of the spinal cord [9, 10]. This is a slow and progressive process, with a fatal outcome. If the cervical segments are involved, it normally leads to paraplegia or tetraparesis/tetraplegia [11]; however, at advanced stages, dogs are unable to feed (dysphagia) dueto the involvement of the cranial nerves [10], and finally require euthanasia.

Breathing difficulties have also been reported $[12,13]$ and were initially studied in German shepherds [14-17] They are also reported in other breeds and in a cat [18]. The typical presentation in dogs $>5$ years of age involves hind limb paresis and ataxia, altered proprioception, and normal spinal reflexes, or in advanced cases, radicular involvement and hyporeflexia. Spinal pain is generally absent. Coexisting disc protrusions can also cause pain during palpation of the spine, masking or complicating the suspicion of subclinical DM. The diagnosis of DM is presumptive in vivo, made by ruling out all other neurological diseases. Genetic testing is usually employed to find a mutation of the alleles of the superoxide dismutase (SOD1) [19, 20], particularly SOD1:c52 A > T, a rare and typical mutation observed in the Bernese breed, and SOD1:c118 G >A, which is found in half-breed and other purebred dogs [21, 22]. The latter is identified by homozygosity for the mutation $(\mathrm{A} / \mathrm{A})$ in $\operatorname{dogs}$ that are considered "affected," whereas the heterozygous (G/A) are identified as "carriers" and those homozygous for the ancestral gene (G/G) as "wild type."

Homozygosity (A/A) seems to predispose dogs to disease development [22] because many dogs with a histopathological confirmation of DM were also homozygous (A/A) for the mutation. However, not all homozygous mutations showed clinical signs of DM due to incomplete penetrance [19]. This could be explained by other predisposing factors, such as environmental effects, epigenetic modification, and longevity. Nonetheless, in heterozygous patients (G/A) or homozygous "wild type" $(\mathrm{G} / \mathrm{G})$ ones with clinical signs of DM [19], definitive diagnosis is only possible by histopathological examination. Neoplasms are the third largest cause of hind limb paresis and ataxia in adult/senior dogs. Depending on whether primitive or metastatic, these could be extradural, intradural/ extramedullary, or intramedullary, and can cause a chronic mass effect directly on the spinal cord or modify vertebral anatomy with subsequent spinal cord adaptation [23, 24]. In WhiteW Terrier [3-25], congenital or acquired malformations and deviations of the spine may result in a chronic compression of the meninges, with consequent interruption of the normal liquor outflow inside the subarachnoid space [26]. This induces meningeal fibrosis with constriction of the spinal cord, reduces spinal cord diameter, and finally results 
in gliosis [27, 28], leading to CM. Therefore, in this study, we aimed to investigate the possible incidence and pathology of $\mathrm{CM}$ in an old and giant dog.

\section{Case Report:}

History: In April 2017, a 11-year-old mixed-breed male neutered dog weighing $39 \mathrm{Kg}$ was referred to us due to slowly progressing weakness of the hind limbs for some months. Anti-inflammatory therapy with carprofen $2 \mathrm{mg} / \mathrm{Kg}$ for 10 days (Rimadylcpr $100 \mathrm{mg}$; Pfizer) was prescribed by the referring veterinarian; however, the treatments did not reduce the symptoms.

Clinical results: The results of the general examination were unremarkable. Neurological examination revealed ataxia and paresis with proprioceptive deficits in the hind limbs. Proprioception of the front limbs was normal. Spinal reflexes were normal, and during palpation of the spine, the dog did not show pain. Neurolocalization T3-L3 was observed. Based on the evaluation of the history and chronic slow progressive course, our differential diagnoses were as follows-

-DM

-Single or multiple disc protrusions.
-Neoplastic spinal cord disease

Diagnostic techniques: Blood exams were unremarkable. The dog was anesthetized with medetomidine (sedastart 1 $\mathrm{mg} / \mathrm{mL}$, all these are without compression of the spinal cord or sub-arachnoidal space highlighted using a contrast medium. Esteve) at a dose of $3 \mathrm{mcgr} / \mathrm{Kg}$, administered with propofol (proposure $10 \mathrm{mg} / \mathrm{mL}$, Merial) at a dose of $4 \mathrm{mg} / \mathrm{Kg}$ and maintained with isoflurane $(2.5 \%)$ /vetflurane (1000 $\mathrm{mg} / \mathrm{g}$, Virbac). The field of view of the first magnetic resonance imaging (MRI) scanners can was very small (14 $\mathrm{cm}$ ). As our patient was large, we first performed a CT myelography (Toshiba Asteion dual slice) of the thoracolumbar spine, by intrathecal inoculation at the A after cerebrospinal fluid (CSF) tapping. The slice thickness was set to $3 \mathrm{~mm}$ using hard and soft tissue filters. The examination was T1 weighted imaging SET1WI (TE 26, TR 800 ms, Nex 3) on sagittal and transverse planes. The CT myelography can 1) and spin-echo completed using MRI (0.2T unit) (Esaote Vet Mr), with turbo spin-echo T2 weighted imagingTSET2WI (TE 80, TR $2600 \mathrm{ms,} \mathrm{Nex} \mathrm{revealed}$ spondylosis of the vertebrae from T4 to T7, with multiple disc protrusions on the first lumbartract (Figure 1A-C).

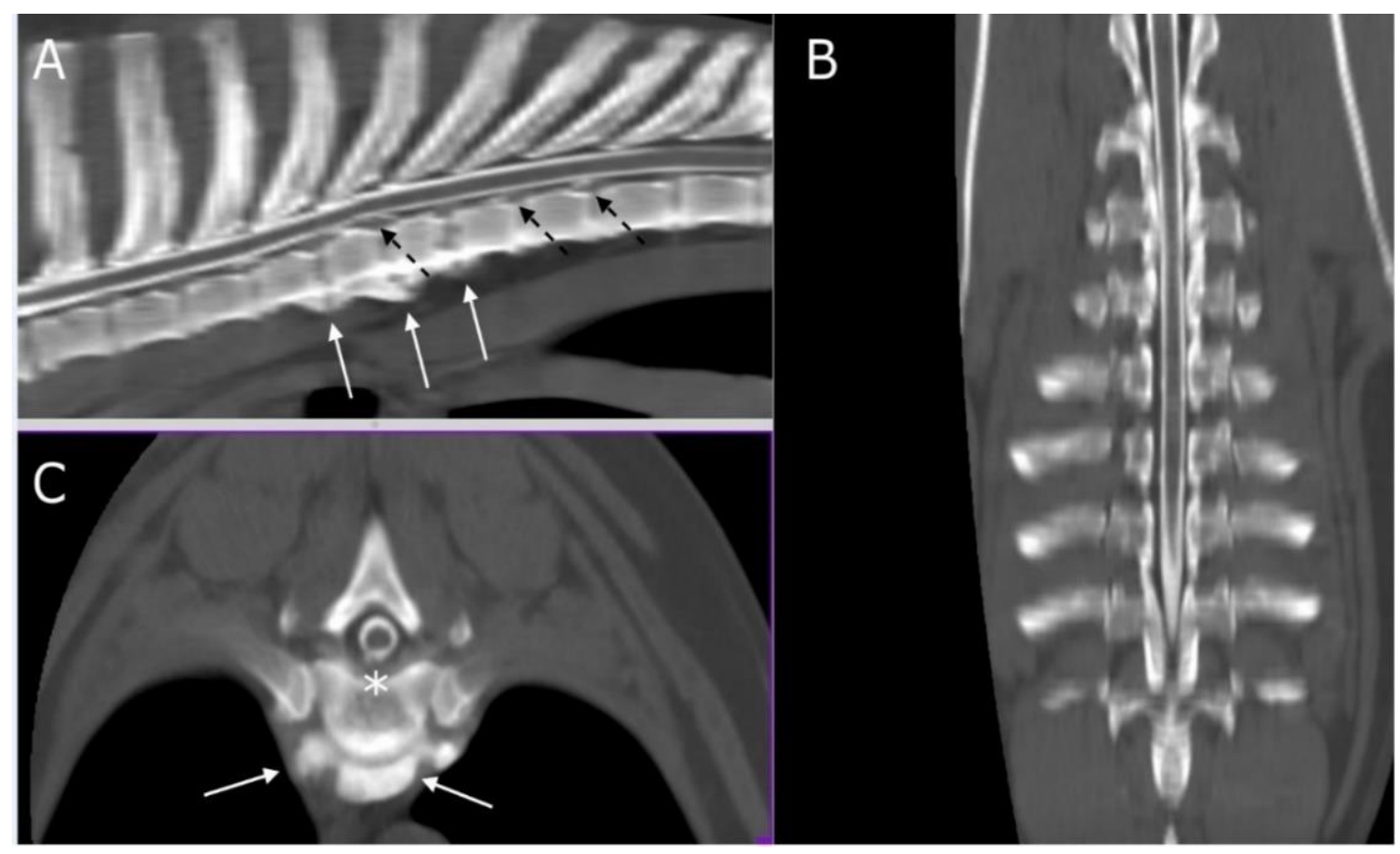

Figure (1): multi-planar reconstruction (MPR) of CT myelography scan on sagittal (A), transverse (B), and dorsal (C) planes. White arrows indicate from T4 up to T7 (A) and at the level of T5-T6 (B) ventral spondylosis. Asterisk shows the disc protrusion in T5-T6 (B) while dashed black arrows (A) indicate multiple disc protrusions in T7-T8 and T8-T9. 
This was confirmed as generalized disc degeneration using MRI (Figure 2), at sites T3-T4, T5-T6, T7-T8, T8-T9, T13L1, and L1-L2, without compressions or signal changes of the spinal cord (Figure 1C, 3B, 4B-C and 5). Widening of the epidural and subarachnoid space was noted (Figure 4 B-C); this alteration was considered secondary to reduced transverse diameter of the spinal cord. CSF was normal on examination. After 23 months, a new MRI was performed using a 0.3T unit (Hitachi Airis II), with turbo spin-echo T2 weighted imaging $(\mathrm{TSET} 2 \mathrm{WI}=\mathrm{TE} 120$, TR 5000, and Nex 4), spin-echo T1 weighted imaging (SET1WI= TE30, TR448, Nex 2), and short tau inversion recovery (STIR= TE 25, TI 110, TR 6000, and Nex 2) in the sagittal and transverse planes, confirming the previous findings.

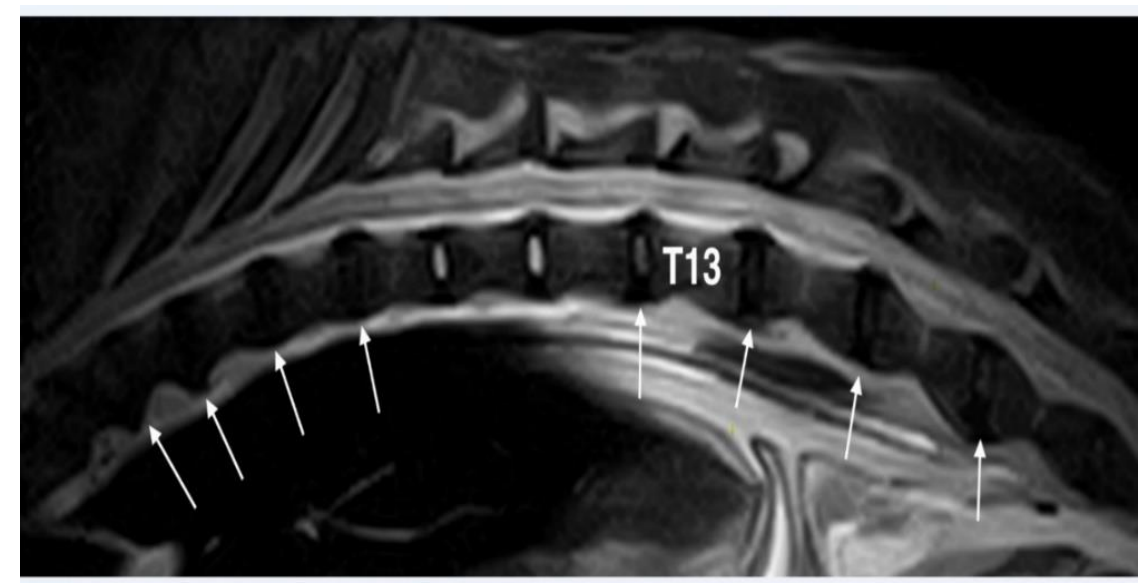

Figure (2): Fast spin-echo (FSE) T2 weighted image of the thoraco-lumbar spine on sagittal plane: With the exception of T10-T11 and T11-T12 discs that seem normal, all others are degenerated (white arrows).

Diagnosis: On diagnosis, widespread spondylosis and multiple disc protrusions without spinal cord compression or signal alterations were observed (Figure 5); however, the diameter was reduced, and the epidural and subarachnoid space were widened. These findings suggested concomitant DM. At that time, the owner refused the genetic test.

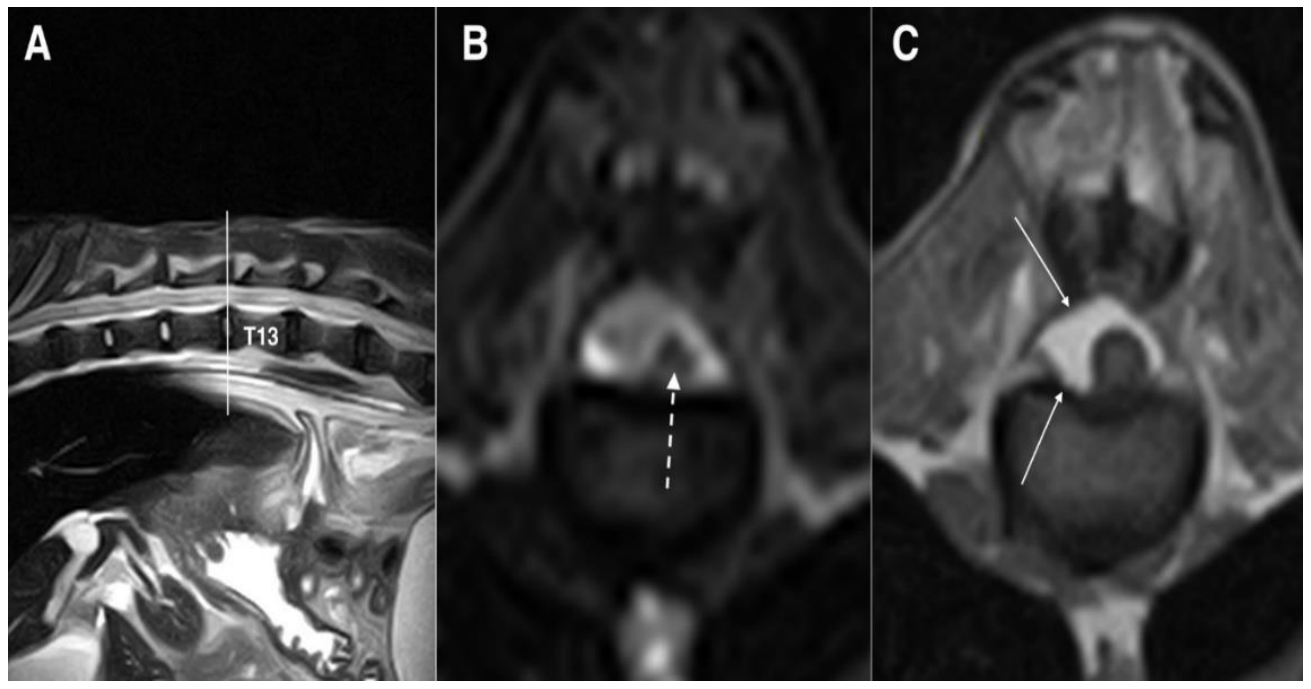

Figure (3): Fast spin-echo (FSE) T2 weighted images on sagittal (A) and transverse (B) planes and SE T1 weighted on transverse (C) plane, at the level of intersomatic space T12-T13: the spinal cord is significantly reduced in diameter and no signal alteration is reported in T2 weighted images (dashed arrow), while the spinal canal is replaced by epidural fat (white arrows) on a T1 weighted image on the right side of the spinal cord 


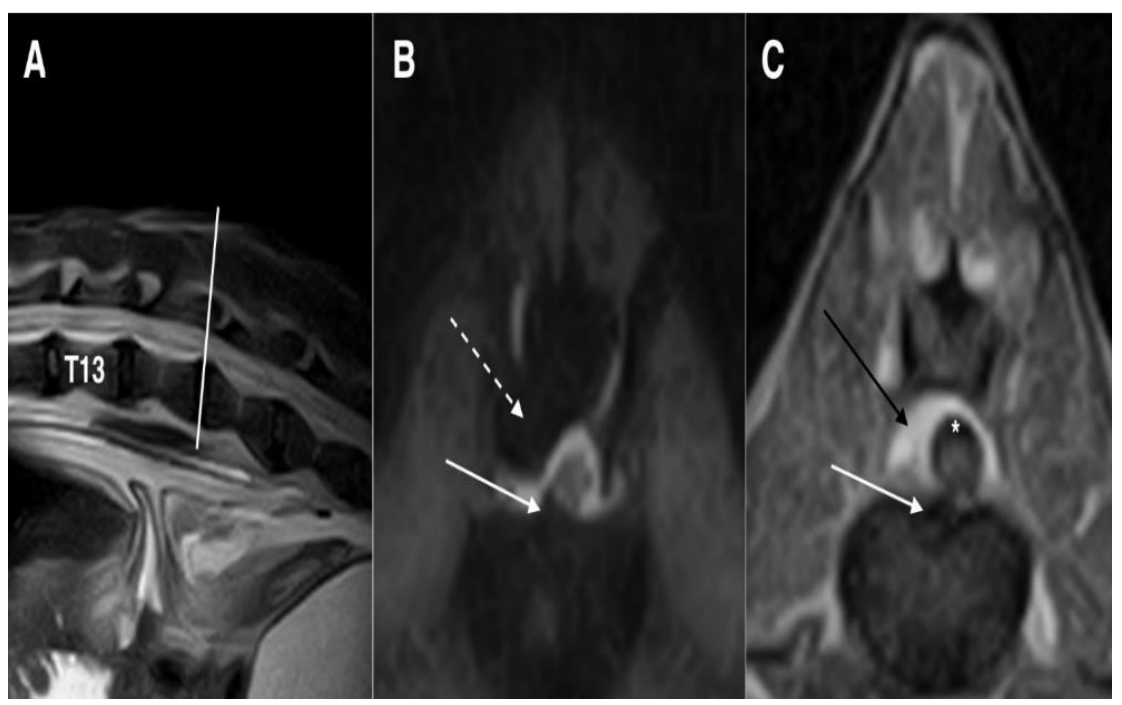

Figure (4): Fast spin-echo (FSE) T2 weighted images on sagittal (A) plane, short tau inversion recovery (STIR) sequence on the transverse (B) plane and $\mathrm{T} 1$ weighted image on the transverse (C) plane at the intersomatic space of L1-L2: white arrows (B and C) show disc protrusion that produces only a deviation of the spinal cord, without compression. Black arrow (C) shows epidural fat that appears hyperintense, while in the STIR sequence (B) the signal is null (dashed arrow). Note that the sub-arachnoidal space appears to be enlarged (asterisk)

Treatment: Due to the absence of significant spinal cord compressions, decompressive surgery was not useful. Further, as reported by the owner, a previous course of antiinflammatory therapy was unsuccessful. Therefore, we did not suggest another treatment cycle, and instead prescribed physiotherapy.
Progress and results: The dog was stable at the first followup after 30 days. The dog presented again seven months later owing to the appearance of a bilateral perineal hernia and mild worsening of the clinical signs. On this occasion, the owner agreed to the genetic test for DM, which revealed homozygosity for the ancestral gene $(\mathrm{G} / \mathrm{G})$.

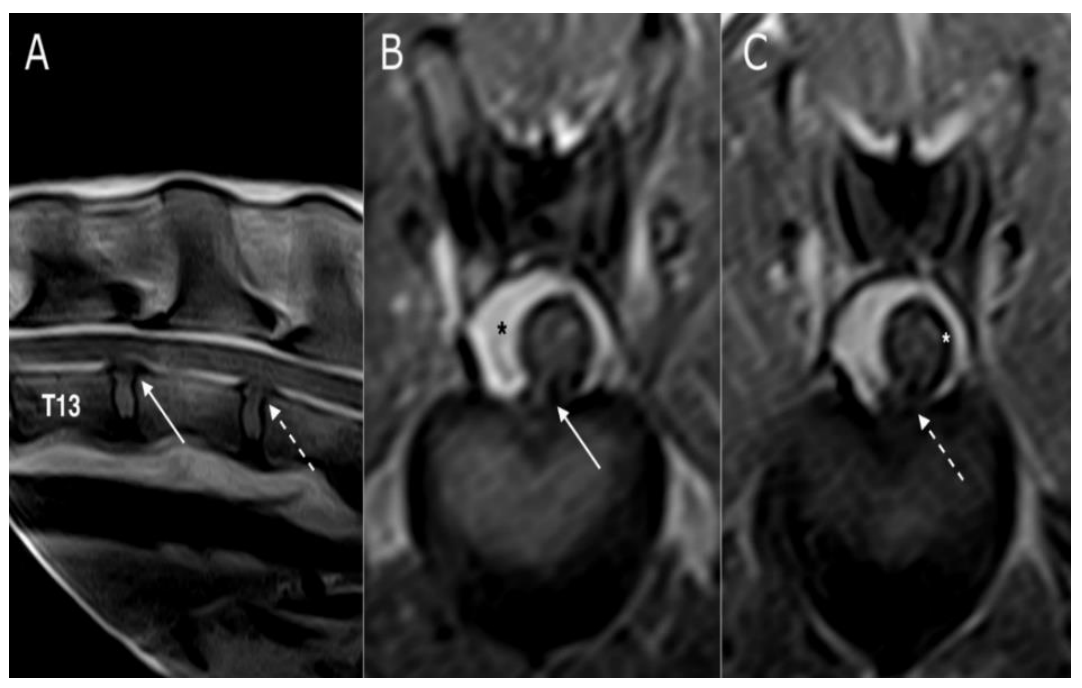

Figure (5): SE T1 weighted images on sagittal (A) and transverse planes (B and C). Disc protrusions in the space T13-L1 (white arrow) and L1-L2 (dashed arrow), without spinal cord compression at both sites (B and C). The black asterisk (B) highlights the epidural fat that replaced the spinal canal, while the white asterisk (C) shows the enlarged subarachnoid space 
After another 16 months, the patient showed worsening hind limb paresis; however, it was still ambulatory. Severe atrophy of the hind limb muscles was also observed. Due to the onset of severe megacolon that was not responsive to medical and dietary treatments, and severe chronic kidney failure, the owner decided to euthanize the animal. A new MRI for diagnostic purposes was performed, and the thoracolumbar spinal cord was collected for histopathological examination.

The MRI scan confirmed the presence of the protrusions already detected in previous imaging studies. Disc protrusions were still low and were not significantly compressive, and the spinal cord had a normal signal; however, the reduced diameter and intra-canal free space were mostly filled with epidural fat ventrally and laterally on the left side of the spinal cord. These findings were confirmed using necropsy (Fig 6A). The segment of the spinal cord between T5 and L7 (Fig 6B) was histopathologically analyzed after fixation in $10 \%$ neutral buffered formalin, followed by paraffin embedding and routine staining of the slides using hematoxylin and eosin.

Macroscopic examination of the entire length of the spinal cord on its ventral surface showed massive osseous metaplasia of the dura mater with evidence of $\mathrm{CM}$, while the inner spinal cord surface was within the normal range. On trimming, white-grey boundaries were well delineated and within the physiological limits. Mild dilation of the ependymal canal was also reported. Microscopic findings revealed concentric enlargement of the meningeal spaces, both subdural and subarachnoid, surrounded by floppy dura mater (Fig 7A) that showed mild to moderate deposition of osteoid matrix and proliferation of osteocytes, rarely comprising osteoblasts (osseous metaplasia) (Fig. 7B). The spinal cord presented with mild, diffuse, bilateral symmetry, edema, and spongiosis of the superficial white matter that frequently featured multiple distended myelin tubes, randomly distributed among large fiber tracts. Nerve roots, mainly originating from the dorsal horn, displayed mild to moderate, multifocal, wide spacing of the nerve fibers (edema) together with mild, multifocal evidence of Wallerian-like degeneration, and rarely included subtle lymphocytic aggregates (Fig. 7C-D). The definitive diagnosis was $\mathrm{CM}$ due to remodeling of the meninges with progressive constriction of the spinal cord. Altered distribution of the mechanical forces among the meningeal layers with stiffening of the ventral pachymeninges was considered a most likely trigger. No evidence of primary degeneration of the spinal cord gray matter, as observed in canine DM, was observed.

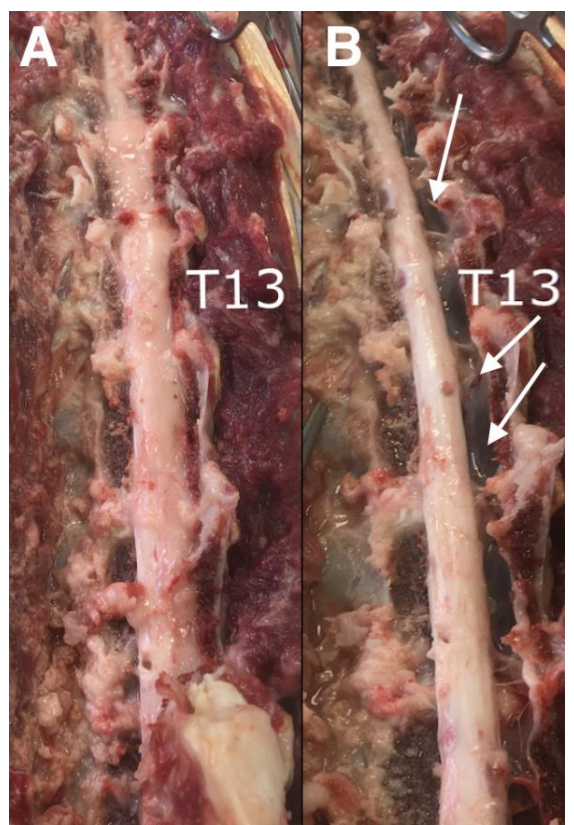

Figure (6): Necroscopy images showing the replacement of spinal canal by the epidural fat (A). After fat removal (B), the reduced diameter of the spinal cord is visible while the spinal canal appears to be empty on the right side (white arrows) as shown in MRI images (see Figures 3,4 , and 5) 


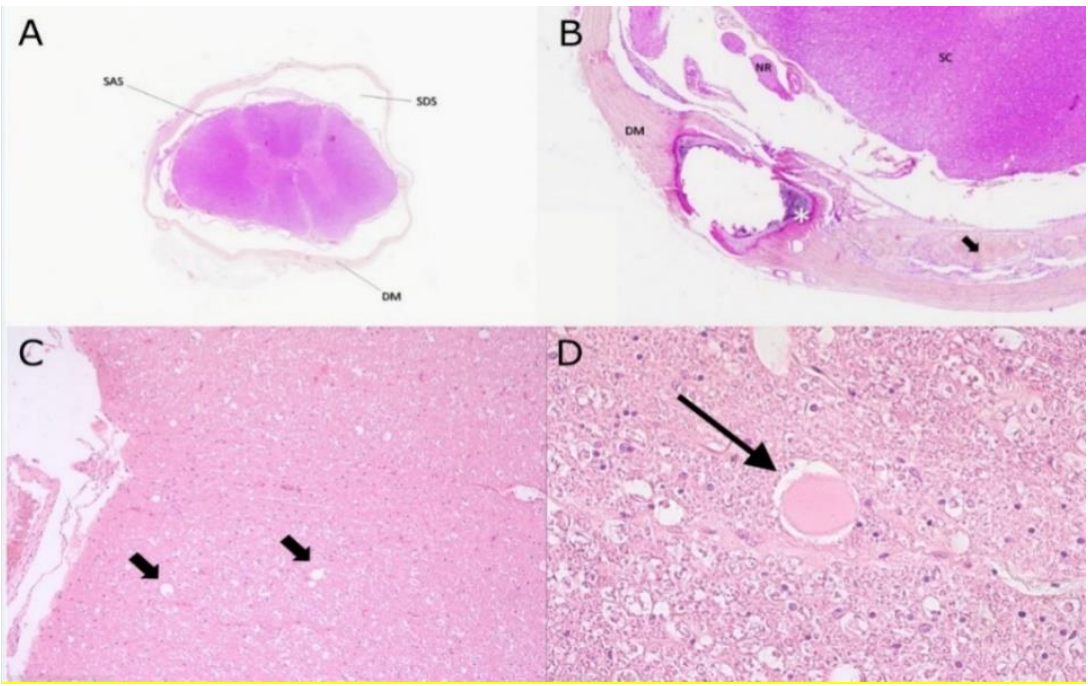

Figure (7): (A) Transverse section of spinal cord featuring concentric thickening and splitting of the dura mater (DM) circumscribing empty fields within the subdural space (SDS). The spinal cord appears overall atrophic with apparent increased volume of the subarachnoid space (SAS). H\&E stain 5x

(B) Marked focal thickening of the dura mater (DM) with osseous metaplasia (asterisk) and splitting accompanied by hyperplasia of meningothelial cells (arrow). Adjacent nerve roots (NR) and spinal cord (SC) are contained within a dilated subdural space. H\&E stain 10x.

(C) Sections of spinal cord white matter presenting with multiple distended myelin tubes along large fibre tracts (arrows) and (D)with axonal spheroid at the center on the right. H\&E 20x and 40x.

\section{Discussion}

Ataxia and paresis are common in giant dogs after 5-6 years of age (Jones et al. 2005), progress slowly, and are associated with hind limb proprioceptive deficits, with normal or sometimes decreased spinal reflexes. In the present report, we describe the condition of a giant dog with moderate and slowly progressive neurological symptoms due to an unexpected degenerative disease of the spinal cord. In fact, the list of differential diagnoses primarily included intervertebral disc degeneration, spondylarthrosis, DM, and neoplasia (Bernardini 2002 'c', Jeffery et al. 2013). In these patients, it is necessary to exclude any other concomitant extra-neurological pathology, parallel or aggravating neurological symptoms (Coates and Wininger 2010); however, advanced imaging is required at a later instance. CT scans permit evaluation of the spinal canal, and myelography can highlight the subarachnoid space. However, MRI offers a better examination of these structures and the spinal cord. Furthermore, genetic testing may be helpful.

With hindsight, we could identify pivotal clinical features that could help reach an "ante-mortem" diagnosis. In our patient, it was important to evaluate discriminating information such as absence of pain during palpation of the spine and lack of response to non-steroidal anti-inflammatory drug (NSAID) treatment $(2 \mathrm{mg} / \mathrm{Kg}$ carprofen for 10 days). It has been reported that pain in subjects with disc protrusions may not be appreciable, and NSAIDs are not effective in dogs with DM. Advanced imaging techniques are fundamental to achieving an ante-mortem diagnosis in these patients. Radiography of the rachis could be performed to detect vertebral malformation or instability; however, a CT scan would be better to evaluate disc extrusions in the chondrodystrophic breed, in whom the extruded material reportedly exhibits hyperattenuation due to chondroid degeneration of discs (Hansen I) (Jefferey et al. 2013). In our dog, we expected fibroid disc degeneration (also Hansen II); therefore, a CT scan with myelography was performed, allowing evaluation of the spinal canal, subarachnoid space, and possible spinal cord compressions. Indeed, suspicion of reduced spinal cord diameter, multiple disc protrusions, and diffuse spondylosis were observed by imaging, which has 
been reported to be useful in these cases (Jones et al. 2005; Levine et al. 2006). A major limitation of this imaging technique in case of multiple protrusions is the need to highlight the most acute lesion responsible for neurological signs, and MRI added value in the diagnosis of disc and/or spinal cord diseases (Henke et al. 2013; Jeffery et al. 2013), confirming the reduction of diameters, low spinal cord compression without signal alterations, and widening of the epidural and subarachnoid space. These findings suggest the coexistence of chronic disc protrusions and DM. The use of NSAIDs in the patient was unsuccessful, and we decided to commence physiotherapy, which is currently considered the gold standard to postpone the inexorable deterioration of clinical signs (Coates and Wininger 2006; Kathmann et al. 2001). Definitive diagnosis of DM can only be made through histologic examination (Awano et al. 2009); however, a genetic test for the mutation of alleles of the gene SOD1 could be useful to reach a presumptive "ante-mortem" diagnosis in mixed breeds (Zeng et al. 2014). In this case, the genetic test was negative for the mutation and the dog remained ambulatory for 23 months. Hence, chronic compression of the spinal cord due to multiple disc protrusions was considered the presumptive ante-mortem diagnosis, and neurological findings were attributed to spinal cord atrophy due to chronic compression, similar to those reported in previous studies (Griffiths 1972; Jeffery et al. 2013). A definitive diagnosis of $\mathrm{CM}$ was made after a histological examination. However, the question regarding this patient could be: what really happened to the spinal cord? Why did it not show any signal alterations with reduced functionality? Although this study reports a single subject, it permits us to advance the hypothesis that chronic compression caused by the disc protrusions involves mechanical stimulation and irritation, leading to pathologic remodeling and consequent stiffening of the lepto- and pachymeninges with progressive constriction of the spinal cord (Fadda et al. 2013) and that the alteration accompanies neurological symptoms. Indeed, in our report, the dog only had a slowly progressive course, and it was not dramatic, as seen in cases of DM where dogs become paraplegic, or as seen in Pugs, where the focal injury of the spinal cord leads to gliosis. In our patient, the spinal cord was reduced in diameter but without any signs of gliosis and the ratio of spinal cord to epidural fat was very close to $50 \%$. This is probably indicative of a very slow adaptation process. Perhaps for the same reason, in contrast to what is reported in Pugs (Driver et al. 2019; Lourinho et al. 2019), our patient did not show urinary or fecal incontinence. In previous reports, spinal cord signal alterations were present (Longo et al. 2019) at MRI imaging with histopathological findings of gliosis (Lourinho et al. 2019) neither of which were found in our patient.

A single case study is a limitation; however, it could explain what happens in dogs with multiple disc protrusions without significant spinal cord compression. Indeed, pachymeninges stiffening can trigger spinal cord compression and consequent atrophy.

\section{Conclusions}

In our patient, multiple disc protrusions with coexistent DM caused neurological deficits, alterations in spinal cord diameters, signal modifications, and enlargement of the epidural space. Although the subject was negative for the SOD1 mutation, perhaps including other predisposing factors could still have indicated DM (Awano et al. 2009). In our case, the negative genetic test finding was accompanied by no typical symptoms of progression (the dog was still ambulatory after 23 months). This led us to conclude that spinal cord atrophy was secondary to chronic compression due to multiple disc protrusions and consequent chronic damage to the spinal cord, as reported in previous studies (Jefferey et al. 2013); however, in this case, CM was diagnosed in a large or giant dog, and this has never been reported before. Obviously, a single report cannot have an absolute value; however, it would be desirable in these patients with a chronic/subtle onset of ambulatory paraparesis, to have a complete clinical and neurological investigation, using imaging, genetic testing, follow-up, and, if possible, performing histological examination of the spinal cord to better characterize the pathology and to evaluate the incidence. If these findings are noted in multiple cases, it could lead to a new differential diagnosis being included for subjects that have all clinical signs of DM even with no evident imaging findings, as in this case, are "wild type" or carriers of the SOD1 mutation, and have only slow and less 
dramatic evolution of the clinical signs, compared to the subjects with confirmed DM.

Acknowledgements: This study could not have been conducted without the support of the entire staff of Centro Veterinario Caleidos who assisted with MRI scanning.

For MRI reporting: Dr. Cristian Falzone, Diagnostica Piccoli Animali, Zugliano, VI, Italy.

For histological examination and immunohistochemistry: Dr. Marco Rosati, Section of Clinical and Comparative Neuropathology, Ludwig-Maximilians-Universität, Munich, Germany.

\section{Declaration of interest: none}

\section{Author contributions}

Category 1:

(a) Conception and design: Del Vecchio

(b) Acquisition of data: Del Vecchio

(c) Analysis and interpretation of data: Del Vecchio

Category 2:

(a) Drafting of the article: Del Vecchio

(b) Revising the article for intellectual content: Del Vecchio Category 3:

(a) Final approval of the completed article: Del Vecchio

Role of the funding source:

This research did not receive any specific grant from funding agencies in the public, commercial, or not-for-profit sectors.

\section{Reference:}

1. Da Costa RC., Moore SA. (2010) Differential diagnosis of spinal diseases. The Veterinary Clinics of North America. Small Animal Practice 40(5): 755-763.

2. Jeffery ND, Levine JM, Olby NJ, Stein VM. (2013) Intervertebral disk degeneration in dogs: consequences, diagnosis, treatment, and future directions. Journal of Veterinary Internal Medicine 27(6): 1318-1333.

3. Fisher SC, Shores A, Simpson ST. (2013) Constrictive myelopathy secondary to hypoplasia or aplasia of the thoracolumbar caudal articular processes in Pugs: 11 cases (1993-2009). Journal of the American Veterinary Medical Association 242(2): 223-229.
4. Rohdin C, Ljungvall I, Häggström J, Leijon A, Lindblad-Toh K, et al. (2020) Thoracolumbar meningeal fibrosis in pugs. Journal of Veterinary Internal Medicine 34(2): 797-807.

5. Ros C, de la Fuente C, de Carellán Mateo AG, Laborda-Vidal P. (2020) Constrictive myelopathy secondary to caudal articular vertebral process dysplasia in West Highland White Terrier dogs. Canadian Veterinary Journal. 61(11): 1155-1158.

6. Bernardini M. (2002) Chapter 15 'c' Patologie del rachide con coinvolgimentoneurologicosecondario. in Neurologia del cane e del gatto. 2nd ed. PolettoEditore. 333-336.

7. Macias C, McKee WM, May C, Innes JF. (2002) Thoracolumbar disc disease in large dogs: a study of 99 cases. Journal of Small Animal Practice. 43(10): 439-446.

8. Gaitero L, Añor S. (2009) Cranial thoracic disc protrusions in three German Shepherd dogs. Veterinary Journal. 182(2): 349-351.

9. Bernardini M. (2002) Chapter 14 'b' Malattie degenerative. in Neurologia del cane e del gatto. 2nd ed. PolettoEditore. 305-306.

10. Coates JR, Wininger FA. (2010) Canine degenerative myelopathy. The Veterinary Clinics of North America. Small Animal Practice. 40(5): 929950.

11. Coates JR, March PA, Oglesbee M, Ruaux CG, Olby NJ, et al. (2007) Clinical Characterization of a Familial Degenerative Myelopathy in Pembroke Welsh Corgi Dogs. Journal of Veterinary Internal Medicine 21(6): 1323-1331.

12. Oyake K., Kobatake Y, Kushida K, Maeda S, Kamishina H. (2016) Changes in respiratory function in Pembroke Welsh Corgi dogs with Degenerative Myelopathy. The Journal of Veterinary Medical Science. 78(8): 1323-1327.

13. Katz ML. (2013) Characterization of intercostal muscle pathology in canine Degenerative Myelopathy: a disease model for amyotrophic lateral sclerosis. Journal of Neuroscience Research. 91(12): 1639-1650. 
14. Averill DR. (1973) Degenerative myelopathy in the aging German Shepherd Dog: clinical and pathologic findings. Journal of the American Veterinary Medical Association. 162(12): 10451051.

15. Braund KG, Vandevelde M. (1978) German Shepherd dog myelopathy - a morphologic and morphometric study. American Journal of Veterinary Research. 39(8): 1309-1315.

16. Romatowski J. (1984) Degenerative myelopathy in a German Shepherd. Modern Veterinary Practice 65(7): 535-537.

17. Seim HB. (1996) Conditions of the thoracolumbar spine. Seminars in Veterinary Medicine and Surgery (Small Animal). 11(4): 235-253.

18. Mesfin GM, Kusewitt D, Parker A. (1980) Degenerative Myelopathy in a cat. Journal of the American Veterinary Medical Association 176(1): 62-64.

19. Awano T, Johnson GS, Wade CM, Katz ML, Coates J.R,et al. (2009) Genome-wide association analysis reveals a SOD1 mutation. in canine Degenerative Myelopathy that resembles amyotrophic lateral sclerosis. Proceedings of the National Academy of Sciences of the United States of America. 106(8): 2794-2799.

20. Tauro A, Rusbridge C. (2017) "Degenerative myelopathy in the nova scotia duck Tolling Retriever.

21. Wininger FA, Zeng R, Johnson GS, Katz ML, Johnson GC, et al. (2011) Degenerative Myelopathy in a Bernese Mountain Dog with a novel SOD1 missense mutation. Journal of Veterinary Internal Medicine 25(5): 1166-1170.

22. Zeng R, Coates JR, Jhonson GC, Hansen L, Awano T, et al. (2014) Breed distribution of SOD1 alleles previously associated with canine degenerative myelopathy. Journal of Veterinary Internal Medicine. 28(2): 512-521.

23. Bernardini M. (2002). Chapter 13 'a' Neoplasie e sindromiparaneoplastiche. in Neurologia del cane e del gatto. 2nd ed.PolettoEditore. 272-279.
24. Dewey CW, da Costa RC. (2016) Chapter 13 John Wiley and sons. 3rd ed Myelopathies in Practical guide to canine and feline neurology. Iowa, USA.

25. Gallant CA. (2020) Constrictive myelopathy in an 11-year-old West Highland terrier dog. Canadian Veterinary Journal. 61(12): 1319-1321.

26. Meren IL, Chavera JA, Alcott CJ, Barker AK, Jeffery ND. (2017) Shunt tube placement for amelioration of cerebrospinal fluid flow obstruction caused by spinal cord subarachnoid fibrosis in dogs. Veterinary Surgery. 46(2): 289-296.

27. Longo S, Gomes SA, Lowrie M. (2019) Previously unreported magnetic resonance findings of subarachnoid fibrosis leading to constrictive myelopathy in a pug. Journal of Small Animal Practice. 60(5): 324.

28. Lourinho F, Holsworth A, MacConnell F, Trevail R, Gonçalves R, et al. (2019) CliniScal Features and magnetic resonance imaging Characteristics of presumptive Constrictive Myelopathy in 26 Pugs. 32nd ECVN-ESVN Symposium. Oral Abstract p. 27 .

29. Jones JC, Inzana KD, Rossmeisl JH, Bergman RL, Wells T, et al. (2005) CT Myelography of the thoraco-lumbar spine in $8 \mathrm{dogs}$ with degenerative myelopathy. Journal of Veterinary Science. 6(4): 341-348.

30. Levine GJ, Levine JM, Walker MA, Pool RR, Fosgate GT. (2006) Evaluation of the association between spondylosis deformans and clinical signs of intervertebral disk disease in dogs: 172 cases (19992000). Journal of the American Veterinary Medical Association. 228(1): 96-100.

31. Henke D, Gorgas D, Flegel T, Vandevelde M, Lang J, et al. (2013) Magnetic resonance imaging findings in dogs with traumatic intervertebral disk extrusion with or without spinal cord compression: 31 cases (2006-2010). Journal of the American Veterinary Medical Association. 242(2): 217-222.

32. Kathmann I, Demierre S, Jaggy A. (2001) Rehabilitationsmassnahmen in der Kleintierneurologie (Rehabilitation methods in small animal 
neurology). Schweiz Arch Tierheilkd. 143(10): 495502.

33. Griffiths IR. (1972) Some aspects of pathology and pathogenesis of the myelopathy caused by disc protrusions in the dog. Journal of Neurology, Neurosurgery, and Psychiatry. 35(3): 403-413.

34. Fadda A, Oevermann A, Vandevelde M, Doherr M.G, Henke D, et al. (2013) Clinical and pathological analysis of epidural inflammation in intervertebral disk extrusion in dogs. Journal of Veterinary Internal Medicine. 27(4): 924-934.

35. Driver CJ, Rose J, Tauro A, Fernandes R, Rusbridge C. (2019) Magnetic Resonance image findings in pug dogs with thoracolumbar myelopathy and concurrent caudal articular process dysplasia. BMC Veterinary Research. 15(1): 182. 